Supporting Information

\title{
Biocompatible Hydrophilic Modifications of Poly(dimethylsiloxane) Using Self-assembled Hydrophobins
}

\author{
Rui Wang, ${ }^{\dagger}$ Yan-Lian Yang, ${ }^{\dagger}$ Ming Qin ${ }^{\ddagger}$ Li-Kai Wang, ${ }^{\ddagger}$ Lei $Y u,{ }^{\S}$ Bin Shao, ${ }^{\S}$ \\ Ming-Qiang Qiao, ${ }^{\S}$ Chen Wang, ${ }^{\dagger}, * X i$-Zeng Feng ${ }^{\dagger, *}$ \\ ${ }^{\dagger}$ National Center for Nanoscience and Technology, Beijing, 100080, P. R. China \\ The Key Laboratory of Bioactive Materials, Ministry of Education, College of Life \\ Science, Nankai University, Tianjin, 300071, P. R. China. \\ ${ }^{\S}$ Institute of Molecular Biology, College of Life Science, Nankai University, Tianjin, \\ 300071, P. R. China.
}

The Process for the Cultivation and Isolation of HFBI.

\section{Strain}

The hydrophobin HFBI-overproducing strain, Trichoderma reesei VTT D-98692, which contains 3 copies of the HFBI gene, was kindly supplied by VTT Biotechnology (Finland).

\section{Cultivation Methods}


The cultivation media used in the experiments contained, in g/L: glucose, 30; peptone, 4.0; yeast extract, $1.0 ; \mathrm{KH}_{2} \mathrm{PO}_{4}, 4.0 ;\left(\mathrm{NH}_{4}\right)_{2} \mathrm{SO}_{4}, 2.8 ; \mathrm{MgSO}_{4} \cdot 7 \mathrm{H}_{2} \mathrm{O}, 0.6$; $\mathrm{CaCl}_{2} \cdot 2 \mathrm{H}_{2} \mathrm{O}, 0.8$ and adjusted $\mathrm{pH}$ to 5.0 before sterilization. The glucose and $\left(\mathrm{NH}_{4}\right)_{2} \mathrm{SO}_{4}$ concentration were kept within the limits of $10-25 \mathrm{~g} / \mathrm{L}$ and $1.0-2.5 \mathrm{~g} / \mathrm{L}$ by addition of sterile $35 \%$ glucose solution and $50 \%\left(\mathrm{NH}_{4}\right)_{2} \mathrm{SO}_{4}$ solution.

\section{Characterizations}

Measurement of biomass was carried out as described. ${ }^{1}$ Glucose was assayed by the method of Fehling test. Nitrogen content was determined with Kjeldahl nitrogen determination method. Tris-Tricine SDS-PAGE was done as described. ${ }^{2}$

\section{Isolation of HFBI from Biomass}

Since most of the HFBI was associated with the fungal mycelium, the pellets of mycelium were used directly for purification of HFBI. Primary isolation of HFBI was carried out as described ${ }^{3}$ and further purification was done with hollow fiber membrane (6000 MW cutoff, Tianjin Motian membrane ENG. \& TECH. Co., Ltd) to remove pigments and salts.

\section{Results}

After fermentation for 5 days, $20.33 \mathrm{~g} / \mathrm{L}$ of the mycelia was obtained and the yield of HFBI in biomass is $1.130 \mathrm{~g} / \mathrm{L}$. Purified HFBI product was performed in Tris-Tricine SDS-PAGE and the result is showed in Figure S1. 


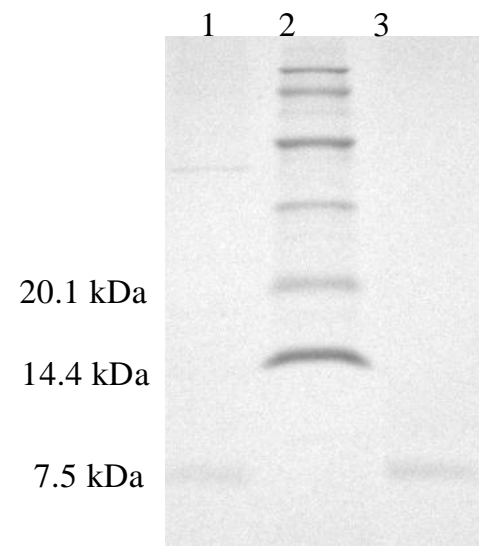

Figure S1. Tris-Tricine SDS-PAGE of HFBI product.

Lane 1: HFBI product isolated from Trichoderma reesei VTT D-98692; Lane 2: protein molecular weight marker-low, Takara; Lane 3: standard solution of HFBI.
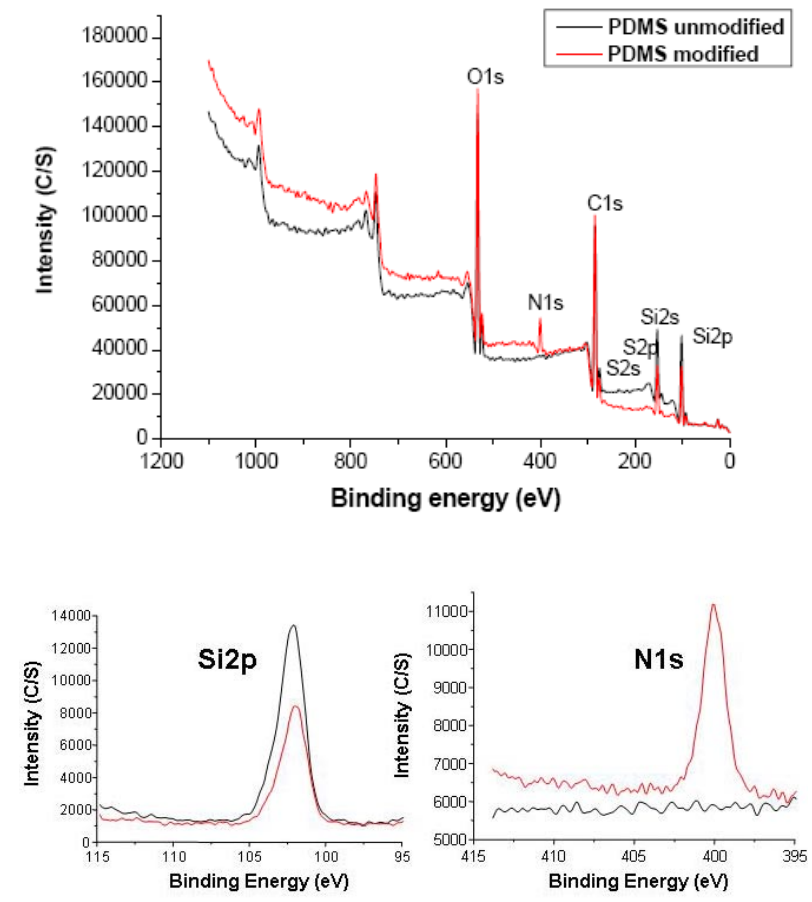

Figure S2. Full XPS spectra and the XPS relative intensities for the Si2p and N1s maxima of the modified and unmodified PDMS substrates. 

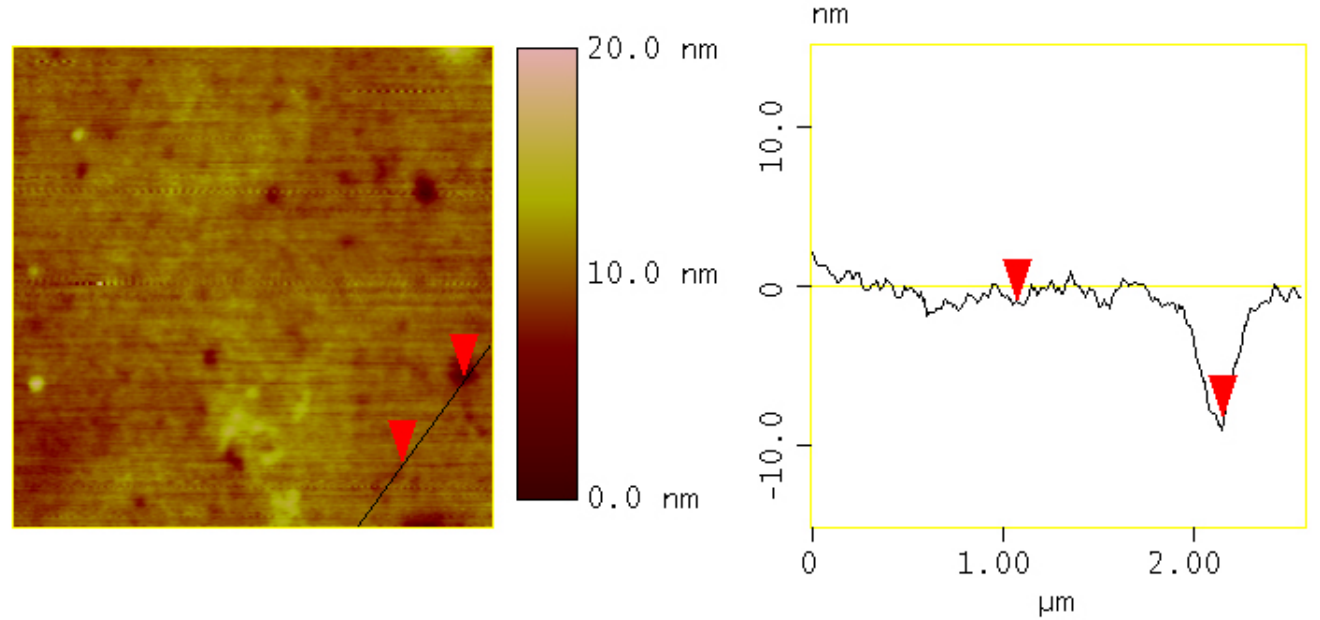

Figure S3. AFM image of HFBI modified PDMS surface (left, scan size: $5 \times 5 \mu \mathrm{m}$ ) and the color scale on the right represents the $\mathrm{z}$ scale of the image. The sectional profile corresponds to the black line in the left image (right).

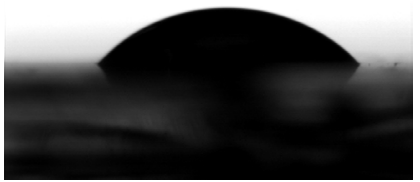

Figure S4. Micrographs of $5 \mu \mathrm{L}$ water droplets on the HFBI modified PDMS substrate after 20 days in air, $\alpha=52.6 \pm 0.7^{\circ}$.

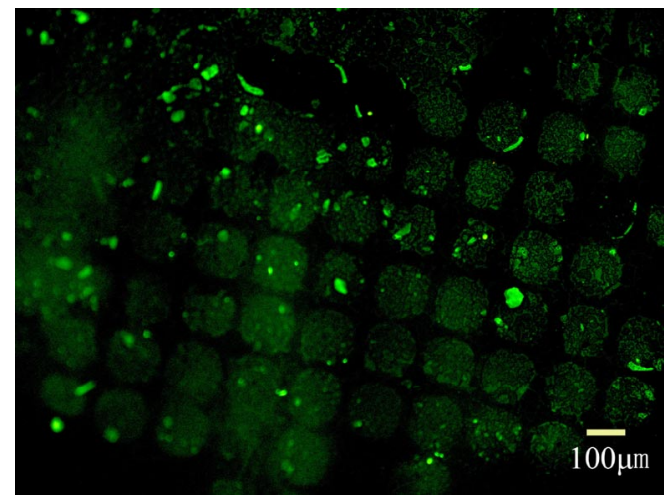

Figure S5. Fluorescent images of patterned chicken IgG on the HFBI modified PDMS 
substrate after 20 days in air.

\section{References:}

1. Bailey, M. J.; Askolin, S.; Horhammer, N. Microbiol. Biotechnol. 2002, 58, 721.

2. Ausubel F. M. Short protocols in molecular biology.

3. Linder, M.; Selber, K.; Setala, T. N. Biomacromolecules 2001, 2, 511. 